\title{
Comparing The Performance of Bayesian And Frequentist Analysis Methods of Irregular Fractional Factorials Using Design Based optimality And Efficiency Criteria
}

\author{
${ }^{1 *}$ Ali H. U., ${ }^{1}$ Lasisi K. E., ${ }^{2}$ Nwaosu S. C. \\ ${ }^{1 *}$ Department of Mathematics, University of Jos, Nigeria \\ ${ }^{l}$ Department of Mathematical Sciences, Abubakar Tafawa Balewa University, P. M. B. 0248, Bauchi, Nigeria. \\ ${ }^{2}$ Department of Mathematics/Statistics,University of Agriculture, Makurdi, Nigeria
}

\begin{abstract}
Irregular fractional factorial designs are widely used in screening experiments. Several analysis methods were proposed for identifying main effects and their interactions in the past twenty years. The goal of this research was to evaluate the performance of two Bayesian and two Frequentist approaches using some real data. The two Bayesian approaches are Bayes screening method and Empirical Bayes method. The two Frequentist approaches are traditional stepwise variable selection method and least angle regression method. The comparison of analysis methods is demonstrated using $A \& D$ optimality and $A \& D$ efficiency, the results obtained showed that the Frequentist approach is the best. We recommend the use of Frequentist approach in analyzing irregular fractional factorial designs at two levels because it works with current data and takes into consideration that variability comes from our sampling.
\end{abstract}

Keywords: Bayesian, Frequentist, Irregular, and Screening.

\section{Introduction}

Screening is the process of discovering, through statistical design of experiments and modeling, those controllable factors or input variables that have a substantive impact on the response or output which is either calculated from a numerical model or observed from a physical process. [1] In many scientific investigations, the main interest is in the study of effects of many factors simultaneously. Factorial designs, especially twolevel or three-level factorial design, are the most commonly used experimental plans for this type of investigation. A full factorial experiment allows all factorial effects to be estimated independently. However, it is often too costly to perform a full factorial experiment, so a fractional factorial design, which is a subset or fraction of a full factorial design, is preferred since it is cost-effective. [2] Irregular two-level fractional factorial designs such as Plackett-Burman designs are becoming increasingly popular choices in many fields of scientific investigation due to their run size economy and flexibility. The run size of irregular two-level factorial design is a multiple of 4 . They fill the gaps left by the regular two-level fractional factorial designs whose run size is always a power of 2 i.e. $4,8,16, \ldots$ In irregular factorial designs each main effect is partially confounded with all the two-factor interactions not involving itself. Because of this complex aliasing structure, irregular factorial designs had not received sufficient attention until recently. [3]

\section{Materials And Methods}

The analysis was carried out using Optex procedure of the SAS software package and R software package.

The Empirical Bayes Method: The empirical work of [21] has demonstrated that empirical Bayes method makes use of likelihood functions and conjugate priors for estimating the variances at high and low levels respectively.

A conjugate prior is given by

$$
f\left(\beta, \sigma^{2}\right)=\frac{\left(\frac{\alpha}{2}\right)^{\frac{d}{2}} \alpha^{2} \frac{(d+p+2)}{2}}{(2 \pi)^{-1 / 2}|V|^{1 / 2} \Gamma\left(\frac{d}{2}\right)} \exp \left\{-\frac{1}{2 \sigma^{2}}\left[(\beta-m)^{1} V^{-1}(\beta-m)\right]+\alpha\right\}
$$

With $\alpha>0, \mathrm{~d}>0, \mathrm{~m} \in \Re^{p}$ and $\mathrm{V}$ is a $\mathrm{p} \times \mathrm{p}$ positive definite matrix. A normal-inverse-gamma distribution.

The Bayes Screening Method: The Bayes screening method proposed by Box and Meyer (1986) [22] use the marginal posterior probability to identify active factors and effects. In the screening approach, models with different combinations of main effects and interactions are created. We consider a set of models 
$M_{0}, \ldots, M_{m}$. Each model $M_{i}$ has a parameter $\beta_{i}$ such that the probability density of response $\mathrm{Y}$ is $\operatorname{Pr}\left(Y / M_{i}, \beta_{i}\right)$. The prior probability of model $M_{i}$ is $\operatorname{Pr}\left(M_{i}\right)$ and the prior probability density of $\beta_{i}$ is $\operatorname{Pr}\left(\beta_{i} / M_{i}\right)$. The predictive density of $Y$ is denoted as

$\operatorname{Pr}\left(Y / M_{i}\right)=\int \operatorname{Pr}\left(Y / M_{i}, \beta_{i}\right) \operatorname{Pr}\left(\beta_{i} / M_{i}\right) d \beta_{i}$ for all $\beta_{i}$

The posterior probability of model $\mathrm{N}_{\mathrm{i}}$ given $\mathrm{Y}$ is then calculated by the following equation:

$$
\operatorname{Pr}\left(M_{i} / Y\right)=\frac{\operatorname{Pr}\left(M_{i}\right) \operatorname{Pr}\left(Y / M_{i}\right)}{\sum_{\text {allj }} \operatorname{Pr}\left(M_{j}\right) \operatorname{Pr}\left(Y / M_{j}\right)}
$$

The Traditional Frequentist Method: This method [24] makes use of the half-normal probability plot to identify significant main effects based on the assumptions of effect sparsity and effect heredity. Effect sparsity is the Pareto Principle in experimental design states that only a small number of effects in the experiment are relatively important. Moreover, effect heredity principle describes that an interaction is active only if at least one of its component main effects is considered as important.

The Least Angle Regression (LARS) Method: This approach [25] considers three levels of heredity principles: no heredity, weak heredity and strong heredity. For the purpose of this work, the strong heredity will be considered since here both of its component factors have to be significant.

Under the strong heredity principle, if an interaction term is selected, the corresponding parent effects must be included. One can calculate the average predictability of all variables that must be included.

III. Results And Discussion

Table (1): A 12-run Plackett-Burman Design generated from Minitap Software

\begin{tabular}{cccccccccccccc}
\hline RUN & A & B & C & D & E & F & G & H & I & J & K & RESPONSE \\
\hline $\mathbf{1}$ & +1 & +1 & -1 & +1 & +1 & +1 & -1 & -1 & -1 & +1 & -1 & 1.635 \\
$\mathbf{2}$ & +1 & -1 & +1 & +1 & +1 & -1 & -1 & -1 & +1 & -1 & +1 & 4.721 \\
$\mathbf{3}$ & -1 & +1 & +1 & +1 & -1 & -1 & -1 & +1 & -1 & +1 & +1 & 2.032 \\
$\mathbf{4}$ & +1 & +1 & +1 & -1 & -1 & -1 & +1 & -1 & +1 & +1 & -1 & 0.328 \\
$\mathbf{5}$ & +1 & +1 & -1 & -1 & -1 & +1 & -1 & +1 & +1 & -1 & +1 & 2.384 \\
$\mathbf{6}$ & +1 & -1 & -1 & -1 & +1 & -1 & +1 & +1 & -1 & +1 & +1 & -1.348 \\
$\mathbf{7}$ & -1 & -1 & -1 & +1 & -1 & +1 & +1 & -1 & +1 & +1 & +1 & 2.982 \\
$\mathbf{8}$ & -1 & -1 & +1 & -1 & +1 & +1 & -1 & +1 & +1 & +1 & -1 & -0.981 \\
$\mathbf{9}$ & -1 & +1 & -1 & +1 & +1 & -1 & +1 & +1 & +1 & -1 & -1 & 2.108 \\
$\mathbf{1 0}$ & +1 & -1 & +1 & +1 & -1 & +1 & +1 & +1 & -1 & -1 & -1 & 1.793 \\
$\mathbf{1 1}$ & -1 & +1 & +1 & -1 & +1 & +1 & +1 & -1 & -1 & -1 & +1 & -3.873 \\
$\mathbf{1 2}$ & -1 & -1 & -1 & -1 & -1 & -1 & -1 & -1 & -1 & -1 & -1 & -5.152
\end{tabular}

\section{A-EFFICIENCY FOR THE FREQUENTIST APPROACH}

$$
\begin{aligned}
A-\text { Efficiency } & =100 \times \frac{1}{N_{D} \text { trace }\left[\left(X^{1} X\right)^{-1}\right] / p} \\
& =100 \times \frac{1}{12 \times 0.9167 / 11} \\
& =100 \times \frac{1}{1.00003636364} \\
& =100 \times 0.9999636377 \\
& =99.9964
\end{aligned}
$$

\section{D-EFFICIENCY FOR THE FREQUENTIST APPROACH}

$$
\begin{aligned}
D-\text { Efficiency } & =100 \times \frac{1}{N_{D}\left|\left(X^{1} X\right)^{-1}\right|^{1 / p}} \\
& =100 \times \frac{1}{12 \times \sqrt[11]{1.2878 \times 10^{-12}}}
\end{aligned}
$$




$$
\begin{aligned}
& =100 \times \frac{1}{12 \times 0.082999815} \\
& =100 \times \frac{1}{0.995997791} \\
& =100 \times 1.004018291 \\
& =100.4018291 \\
& \approx 100.402
\end{aligned}
$$

$A-$ optimality $=\operatorname{trace}\left(X^{1} X\right)^{-1}=0.9167$

$D$-optimality $=\left|\mathrm{X}^{1} X\right|=7.4301 e+11$

\section{A-OPTIMALITY FOR THE FREQUENTIST APPROACH}

$A-$ optimality $=\operatorname{trace}\left(X^{1} X\right)^{-1}=0.9167$

\section{D-OPTIMALITY FOR THE FREQUENTIST APPROACH}

$D$-optimality $=\left|\mathrm{X}^{1} X\right|=7.4301 e+11$

\section{D-EFFICIENCY FOR THE BAYESIAN APPROACH}

The Optex procedure of the SAS software was used to obtain the efficiencies and optimalities and results presented thus;

Bayesian D-efficiency, $\xi_{1}(X)=\frac{\left|I\left(X, \beta_{\text {true }}\right)\right|}{I\left(X_{D-\text { opt }}, \beta_{\text {true }}\right)}$

$$
=99.893
$$

\section{A-EFFICIENCY FOR THE BAYESIAN APPROACH}

Bayesian A-efficiency,

$$
\xi_{2}(X)=\frac{\operatorname{tr}\left(X, \beta_{\text {true }}\right)}{I\left(X_{A-\text { opt }}, \beta_{\text {true }}\right)}
$$

$$
=99.406
$$

\section{D-OPTIMALITY FOR THE BAYESIAN APPROACH}

Bayesian D-optimality,

$$
\begin{aligned}
& \phi_{1}(X)=\operatorname{det}\left[X^{I} X+R\right] \\
& =3.9688 e+011
\end{aligned}
$$

\section{A-OPTIMALITY FOR THE BAYESIAN APPROACH}

Bayesian A-optimality,

$$
\begin{aligned}
& \phi_{2}(X)=-\operatorname{tr}\left[\left(X^{I} X+R\right)^{-1}\right] \\
& =-1.041
\end{aligned}
$$

Table (2): The Optex Procedure results for Bayesian Efficiencies

\begin{tabular}{llll}
\hline Standard Error & Design Number & D-efficiency & A-efficiency \\
\hline $\mathbf{1 . 0 0 6}$ & 1 & $99.893^{*}$ & $99.406^{*}$ \\
$\mathbf{1 . 0 6 7}$ & 2 & 98.845 & 88.410 \\
$\mathbf{1 . 0 9 7}$ & 3 & 98.405 & 83.565 \\
$\mathbf{1 . 0 9 7}$ & 4 & 98.405 & 83.558 \\
$\mathbf{1 . 1 0 3}$ & 5 & 98.234 & 82.770 \\
$\mathbf{1 . 1 2 2}$ & 6 & 98.119 & 79.911 \\
$\mathbf{1 . 1 1 6}$ & 7 & 98.093 & 80.750 \\
$\mathbf{1 . 1 0 3}$ & 8 & 98.060 & 82.627 \\
$\mathbf{1 . 1 0 3}$ & 9 & 98.060 & 82.627 \\
\hline
\end{tabular}


Comparing The Performance Of Bayesian And Frequentist Analysis Methods Of Irregular

\begin{tabular}{llll}
\hline $\mathbf{1 . 1 0 9}$ & 10 & 97.999 & 81.865 \\
\hline
\end{tabular}

Table (3): Results Of Analysis

Summary of results

\begin{tabular}{lllll}
\hline Method & A-efficiency & D-efficiency & A-optimality & D-optimality \\
\hline Frequentist & 99.996 & 100.402 & 0.9167 & $7.4301 \mathrm{e}+11$ \\
Bayesian & 99.406 & 99.893 & -1.041 & $3.9688 \mathrm{e}+011$ \\
\hline
\end{tabular}

\section{Discussion}

From table (3) above, the values of A-efficiency and D-efficiency for the Frequentist approach are higher compared with the Bayesian approach, this shows that the Frequentist is more efficient.

From table (3) above, the values of A-optimality and D-optimality for the Frequentist approach are higher when compared with the Bayesian approach, and this showed that the Frequentist approach is a better method of analyzing irregular fractional factorial designs.

This work has successfully compared the Bayesian and Frequentist methods of analyzing irregular fractional factorial designs using the designed based optimality criteria at two levels. According to the numerical results obtained using SAS, Minitap and SPSS statistical software, we infer that:

* The Frequentist approach is more A-optimal than the Bayesian approach in the analysis of irregular fractional factorial designs at two levels as shown above.

* The Frequentist approach is more D-optimal than the Bayesian approach in the analysis of irregular fractional factorial designs at two levels as shown above.

* The Frequentist approach is more A-efficient than the Bayesian approach in the analysis of irregular fractional factorial designs at two levels as shown above.

* The Frequentist approach is more D-efficient than the Bayesian approach in the analysis of irregular fractional factorial designs at two levels.

\section{Conclusion}

In this work, we have successfully discussed the two Bayesian methods: Bayes screening and Empirical Bayes methods, and applied them to a two level Plackett-Burman design of 12-runs. We also discussed the two Frequentist methods we considered in this work: Least Angle Regression and the Traditional Frequentist approaches and applied them to a two level Plackett-Burman design of 12-runs. Bayesian approaches are always straightforward but complex and difficult to understand. Furthermore, they are not flexible. The Empirical Bayes method is more flexible and efficient than the Bayes screening method as the empirical Bayes method considers additional priors and the model space in more detail. It was used to obtain the estimates of the responses. The LARS approach is a fast and efficient strategy to identify important main effects. This approach is a good choice to deal with data sets with a large number of factors. Based on the analysis we performed, the results of A \& D optimality as well as A \& D efficiency showed that the Frequentist approach is a better method of analyzing a 12-run irregular fractional factorial designs at two levels.

The Frequentist approach is more practical and logical since it seems reasonable that these events have a specific probability and that the variation is in our sampling. More so, most data analysis from studies are usually done using the Frequentist approach (i.e. confidence interval, hypothesis testing with p value) since it is easily understandable. Hence, when one is using a Frequentist method the experimenter is making predictions on underlying truths of the experiment using only data from the current experiment. We recommend the use of Frequentist approach in analyzing irregular fractional factorial designs at two levels because it believes in working with current data and takes into consideration that variability comes from our sampling.

From figure 1 below, we observed that series of lambda were set from 0 to 1 with the corresponding crossvalidated mean squares error, it was observed that lambda L1-norm between 0.6 to 0.8 proved to be the best with the minimum mean squares error. The lasso algorithm automatically chose the lambda L1-norm parameters to shrink the parameters towards zero due to the presence of multicollinearity in the covariates A to K. and figure 2 also confirm the result of figure 1. In figure 3, it was observed that factor $\mathrm{G}$ has the least probability whereas factor D has the highest probability, their contributions are in order of the probability.

\section{References}

[1]. Box, G.E.P. \& Meyer, R.D. (1993): Finding the Active Factors in Fractionated Screening Experiments; Journal of Quality Technology.

[2]. Shek, Y. W. (2012): Comparison of Analysis Methods for Non-regular Fractional Factorial Designs; University of California, Los Angeles.

[3]. Woods, D. C. \& Lowis S. M. (2015): Model Selection via Bayesian information capacity designs for generalized linear models; Technometrics.

[4]. Jaynes, J., Ding, X., Xu, H., Wong, W.K., \& Ho, C.M. (2013). Application of Fractional Factorial Designs to Study Drug Combinations. Statistics in Medicine, 32, 307-318. 
[5]. Hongquan, Xu, Frederick K. H. Phoa \& Weng Kee Wong (2009): Recent Developments in Nonregular Fractional Factorial Designs; Statistics Surveys. University of California, Los Angeles.

[6]. Hamada, M \& Wu, C.F.J. (2000): Analysis of Designed Experiments With Complex Aliasing; Journal of Quality Technology.

[7]. Ke, Yao. (2008): Selection of Non-Regular Fractional Factorial Designs When Some Two-Factor Interactions are Important; South Dakota State University.

[8]. Phoa, F. K. H., Xu, H. \& Wong, W. K. (2009). The use of nonregular fractional factorial designs in combination toxicity studies. Food and Chemical Toxicology, 47, 2183-2188.

[9]. Ying Zhang. (2006): Bayesian D-Optimal Design for Generalized Linear Models; Blacksburg, Virginia.

\section{Figures}

Figure 1: The Least Angle Regression plot showing Mean Square Error.

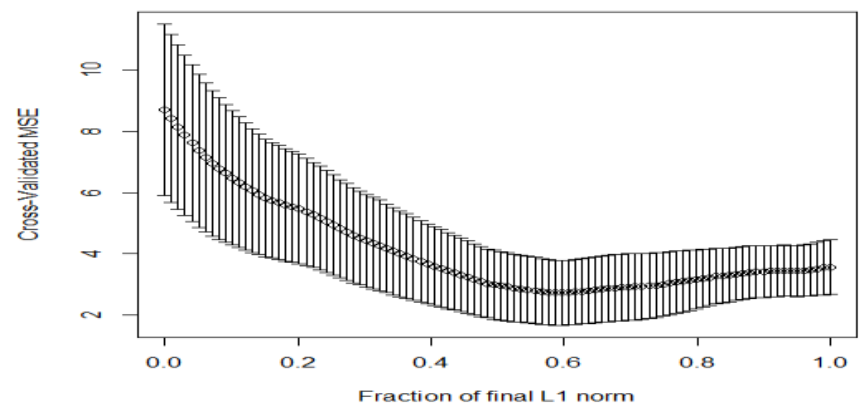

Figure 2: The Bayesian plot showing the Mean Square Error.

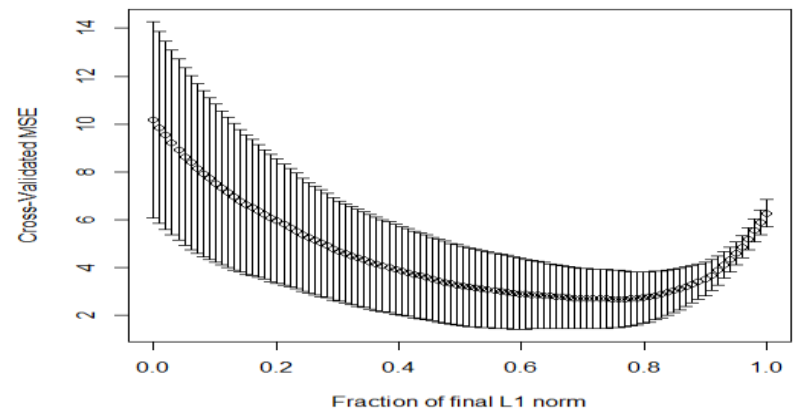

Figure 3: The screening posterior probability plot with all main effects

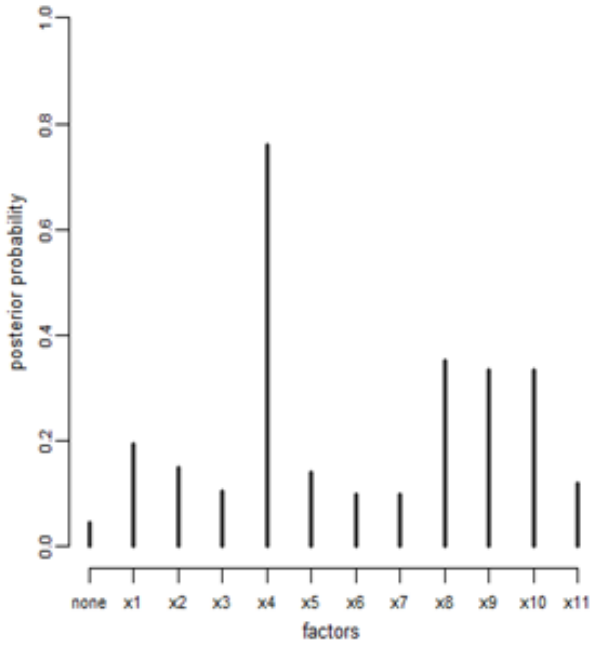

Ali H. U. "Comparing The Performance of Bayesian And Frequentist Analysis Methods of Irregular Fractional Factorials Using Design Based optimality And Efficiency Criteria." IOSR Journal of Mathematics (IOSR-JM) 13.3 (2017): 68-72. 